\title{
Une éclosion de COVID-19 associée à un centre d'entraînement physique en Saskatchewan : leçons pour la prévention
}

\author{
Maureen Anderson ${ }^{1,2 *}$, Ashok Chhetri ${ }^{1,2}$, Edith Halyk ${ }^{3}$, Amanda Lang ${ }^{4}$, Ryan McDonald ${ }^{4}$, \\ Julie Kryzanowski ${ }^{1,5}$, Jessica Minion ${ }^{4}$, Molly Trecker ${ }^{2}$
}

\section{Résumé}

Contexte : Une éclosion de la maladie à coronavirus 2019 (COVID-19) est survenue en Saskatchewan du 12 septembre au 20 octobre 2020. L'événement index, la fréquentation d'un centre d'entraînement physique local, a donné naissance à six éclosions/agrégats de cas supplémentaires dans de multiples contextes. II s'agissait d'une école secondaire, d'un hôpital, de trois lieux de travail ( $A, B$ et $C$ ) et de plusieurs ménages. L'aggrégat comprenait 63 cas au total, soit 27 membres du centre d'entraînement et 36 autres cas de deuxième, troisième et quatrième génération.

Méthodes : Tous les cas de COVID-19 liés à l'éclosion et confirmés en laboratoire ont été inclus dans l'analyse. Les autorités locales de santé publique ont interrogé tous les cas et les contacts et ont mené des enquêtes environnementales dans le centre d'entraînement physique. Nous avons utilisé des méthodes épidémiologiques descriptives pour comprendre la dynamique de transmission de l'aggrégat associé au centre d'entraînement en utilisant l'enquête des cas, l'enquête sur les contacts et les données de laboratoire, y compris le séquençage du génome entier.

Résultats : Les données de séquençage ont confirmé la lignée unique des cas liés à l'aggrégat ( $n=32$ séquencés; coronavirus du syndrome respiratoire aigu sévère 2 [SRAS-CoV-2] lignée B.1.1.72). En plus de la fréquentation du centre d'entraînement, des cas infectieux fréquentaient l'école secondaire et étaient impliqués dans d'autres activités. Malgré la transmission continue dans le centre d'entraînement, aucun cas secondaire n'a été identifié dans l'école secondaire où quatre élèves appartenant à l'aggrégat ont assisté à des cours pendant leur période infectieuse.

Conclusion : Nous décrivons une éclosion de COVID-19 où le ou les cas index fréquentaient un centre d'entraînement, et où la propagation s'est poursuivie pendant 38 jours malgré le dépistage et l'isolement des cas positifs au cours de cette période. En raison de la fréquentation du centre d'entraînement au fil du temps, la fermeture à court terme et le nettoyage peuvent ne pas interrompre les chaînes de transmission. Une mesure de santé publique ciblée et préventive dans les installations d'entraînement physique peut être justifiée. Les mesures de contrôle ont permis de limiter la propagation dans les écoles.
Cette oeuvre est mise à la disposition selon les termes de la licence internationale Creative Commons Attribution 4.0

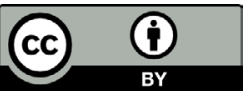

Affiliations

${ }^{1}$ Département de santé communautaire et d'épidémiologie, Université de la Saskatchewan, Saskatoon, SK

2 Saskatchewan Health Authority, santé de la population,

Saskatoon, SK

${ }^{3}$ Saskatchewan Health Authority, services de santé publique, Yorkton, SK

${ }^{4}$ Laboratoire provincial Roy Romanow, Regina, SK

${ }^{5}$ Direction générale de la santé de la population, ministère de la Santé de la Saskatchewan, Regina, SK

*Correspondance :

maureen.anderson@usask.ca

Citation proposée : Anderson M, Chhetri A, Halyk E, Lang A, Kryzanowski J, Minion J, Trecker M. Une éclosion de COVID-19 associée à un centre d'entraînement physique en Saskatchewan : leçons pour la prévention. Relevé des maladies transmissibles au Canada 2021;47(11):538-44. https://doi.org/10.14745/ccdr.v47i11a08f

Mots-clés : COVID-19, SRAS-CoV-2, centre d'entrainement physique, ventilation, distanciation physique, enquête par aggrégats, séquençage du génome entier, dépistage actif, santé publique

\section{Contexte}

En septembre 2020, les agents de santé publique en Saskatchewan ont observé une augmentation du nombre de cas confirmés en laboratoire de la maladie à coronavirus 2019
(COVID-19) dans la ville $X$. Cinq cas signalés sur une période de deux jours avaient un lien commun avec un centre d'entraînement physique local. La date d'apparition du cas 
index était le 12 septembre, et une éclosion a été déclarée le 27 septembre. Un total de 63 cas liés à l'éclosion ont été identifiés avec des dates d'apparition allant du 12 septembre au 20 octobre 2020. Le lien entre les cas a été confirmé par le séquençage du génome entier. La majorité $(79,4 \%)$ des cas liés à l'aggrégat étaient âgés de 18 à 64 ans, et 54,0\% s'identifiaient comme étant des hommes; tous les cas se sont rétablis. Les cas secondaires $(n=23)$ étaient en grande partie des contacts familiaux et étroits de personnes fréquentant le centre d'entraînement.

Des éclosions de COVID-19 dans des centres d'entraînement/ salles de sport sont survenues dans de nombreuses juridictions tout au long de la pandémie. Les centres d'entraînement sont des lieux à haut risque qui facilitent la transmission du coronavirus du syndrome respiratoire aigu sévère 2 (SRAS-CoV-2). Les chercheurs supposent que plusieurs facteurs de risque contribuent à la transmission virale dans les centres d'entraînement :

- Le temps que les individus passent habituellement dans un centre d'entraînement (environ 50 minutes)

- L'augmentation de la respiration pendant l'activité physique

- La charge virale de la personne infectée

- La taille et la ventilation de l'installation

- Le risque accru associé aux séances d'exercices en groupe, en particulier lorsque les participants et instructeurs sont très proches les uns des autres ou parlent fort par-dessus la musique (1-6)

Il convient de noter que les études analytiques examinant le risque d'infection communautaire par le SRAS-CoV-2 et la fréquentation de centres d'entraînement n'ont pas systématiquement mis en évidence une augmentation de I'acquisition de la maladie chez les personnes fréquentant un centre d'entraînement. Par exemple, une étude menée à Oslo, en Norvège, a réparti de manière aléatoire les individus entre ceux qui avaient accès à un centre d'entraînement et ceux qui n'avaient pas accès à un centre d'entraînement. Les taux de positivité des tests entre les deux groupes après une période de 14 jours ne différaient pas de façon considérable (zéro cas dans le groupe sans accès; un cas non acquis dans le centre d'entraînement dans le groupe avec accès) (7). Une autre étude cas-témoins récente a révélé que, même après ajustement des facteurs de confusion potentiels, la fréquentation d'un centre d'entraînement dans les 14 jours précédant l'apparition de la maladie ne différait pas de manière statistiquement significative entre les personnes symptomatiques dont le test de dépistage de l'infection par le SRAS-CoV-2 était positif et celles dont le test était négatif (8).

La santé publique effectue un travail minutieux lorsqu'elle enquête sur des agrégats de maladies afin d'atténuer la propagation et de prévenir de futures éclosions. En particulier lorsque l'enquête porte sur un nouvel agent pathogène en contexte de pandémie, des enquêtes approfondies sur les agrégats peuvent servir à fournir des données importantes pour comprendre la propagation et le risque d'acquisition. Les objectifs de notre enquête sur l'éclosion étaient d'identifier et d'isoler tous les cas infectieux de SRAS-CoV-2 afin de prévenir toute transmission supplémentaire et de comprendre les conditions sous-jacentes qui ont pu contribuer à la transmission virale dans le centre d'entraînement, en fournissant des données pour des mesures préventives.

\section{Méthodes}

Le laboratoire provincial Roy Romanow utilise des tests d'amplification en chaîne par polymérase avec transcription inverse (RT-PCR) pour identifier la présence du SRAS-CoV-2 dans tous les échantillons nasopharyngés soumis. En vertu de la Public Health Act, 1994, tous les cas d'infection au SRAS-CoV-2 confirmés en laboratoire doivent être signalés aux autorités locales de santé publique. Par le biais d'entretiens individuels, en utilisant une fiche de collecte de données standardisée, les autorités locales recueillent des informations sur les caractéristiques démographiques du cas, la date d'apparition des symptômes et toutes les activités entreprises par le cas pendant la période infectieuse.

Dans cette enquête, des infirmières de santé publique formées ont mené des entretiens de recherche de contacts pour tous les contacts proches nommés par les cas. Les cas devaient s'auto-isoler pendant 14 jours à partir de la date du dernier contact avec un cas confirmé afin de rompre les chaînes de transmission subséquentes. De plus, tous les contacts proches se sont vus proposer un test de dépistage. Un cas a été défini comme un cas de COVID-19 confirmé en laboratoire avec apparition des symptômes le 12 septembre 2020 ou plus tard, présentant un lien épidémiologique au centre d'entraînement. Les cas présentant un lien épidémiologique ont soit fréquenté le centre d'entraînement en personne, soit été identifiés comme faisant partie d'une chaîne de transmission liée à une personne fréquentant le centre d'entraînement.

Nous avons extrait les données de laboratoire du système d'information de laboratoire provincial, un référentiel de données de tous les résultats de laboratoire. Le Laboratoire national de microbiologie du Canada a fourni des données de séquençage du génome entier pour tous les spécimens liés à l'éclosion.

Nous avons utilisé l'épidémiologie descriptive (dénombrements, taux, proportions, courbe épidémique) pour comprendre la charge et le moment de la maladie dans les aggrégats index et associées et pour caractériser les cas et leurs résultats. Nous avons utilisé à la fois des données détaillées des enquêtes de cas et de'aggrégats pour créer un visuel de l'aggrégat index et de la transmission aux autres aggrégats (figure 1). Les données de séquençage du génome entier ont permis de vérifier si les cas 
associés à l'aggrégat étaient liés. Des informations contextuelles qualitatives supplémentaires fournies par des enquêteurs de la santé publique, notamment des infirmières et des inspecteurs de la santé publique, ont été incluses dans l'analyse descriptive.

Figure 1 : Visualisation de l'aggrégat index et de la propagation aux autres aggrégats

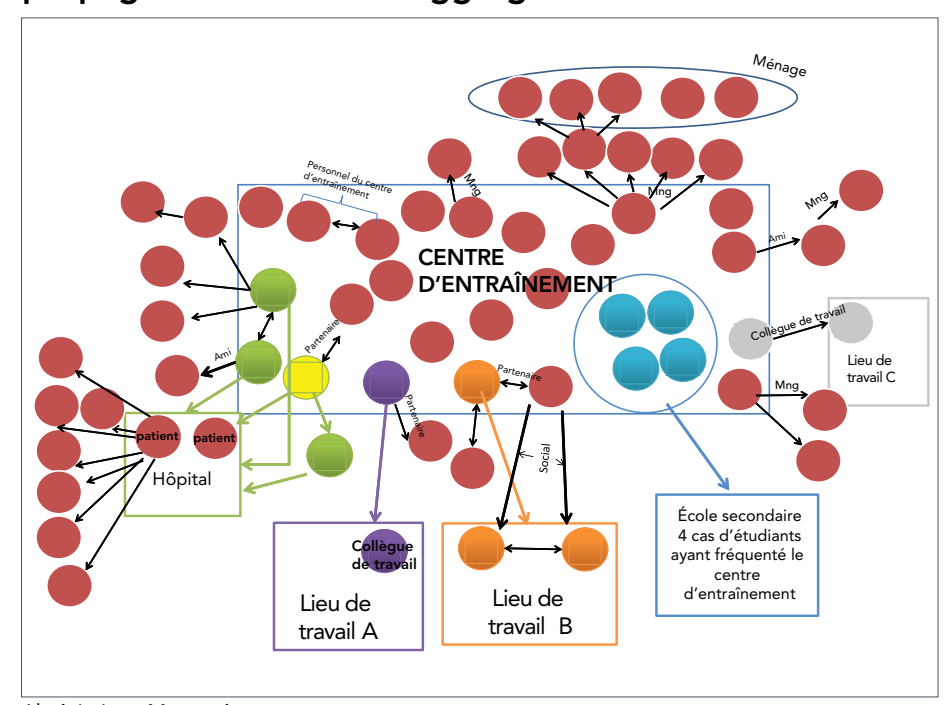

Abréviation : Mng, ménage

\section{Résultats}

La majorité $(79,4 \%)$ des 63 cas liés à l'aggrégat étaient âgés de 18 à 64 ans, et 54,0\% étaient des hommes. Un peu plus de la moitié $(57,1$ \%) n'étaient pas membres du centre d'entraînement. Les cas secondaires étaient en grande partie des contacts familiaux. Aucun cas n'a été hospitalisé ou n'est décédé (voir le tableau 1 pour une description des cas associés à l'éclosion; voir la figure 2 pour une courbe épidémique montrant les données des cas par date d'apparition des symptômes).

Tableau 1 : Épidémiologie descriptive des cas de COVID-19 découlant de la fréquentation d'un centre d'entraînement comme exposition index, ville $X$, Saskatchewan, du 12 septembre au 20 octobre 2020 $(n=63)$

\begin{tabular}{|l|l|r|r|}
\hline \multicolumn{1}{|c|}{ Variable } & \multicolumn{1}{c|}{ Catégorie } & \multicolumn{1}{c|}{$\%$} \\
\hline \multirow{4}{*}{ Âge } & Enfants (moins de 18 ans) & 8 & 12,6 \\
\cline { 2 - 4 } & Adultes (18 à 64 ans) & 50 & 79,4 \\
\cline { 2 - 4 } & Personnes âgées (65 ans et plus) & 5 & 7,9 \\
\hline \multirow{2}{*}{ Sexe } & Homme & 34 & 54,0 \\
\cline { 2 - 4 } & Femme & 29 & 46,0 \\
\hline $\begin{array}{l}\text { Membre } \\
\text { du centre } \\
\text { d'entraînement }\end{array}$ & Oui & 27 & 42,9 \\
\cline { 2 - 4 } & Non & 36 & 57,1 \\
\hline $\begin{array}{l}\text { Contexte de } \\
\text { l'aggrégat } \\
\text { associé }\end{array}$ & École secondaire & 4 & 6,3 \\
\cline { 2 - 4 } & Hôpital & 13 & 20,6 \\
\cline { 2 - 4 } & Lieu de travail A & 2 & 3,2 \\
\hline
\end{tabular}

Tableau 1 : Épidémiologie descriptive des cas de COVID-19 découlant de la fréquentation d'un centre d'entraînement comme exposition index, ville $X$, Saskatchewan, du 12 septembre au 20 octobre 2020 ( $\mathrm{n}=63$ ) (suite)

\begin{tabular}{|c|c|c|c|}
\hline Variable & Catégorie & $\mathbf{n}$ & $\%$ \\
\hline \multirow{2}{*}{$\begin{array}{l}\text { Contexte de } \\
\text { l'aggrégat } \\
\text { associée (suite) }\end{array}$} & Lieu de travail B & 3 & 4,8 \\
\hline & Lieu de travail C & 2 & 3,2 \\
\hline \multirow{3}{*}{ Type de cas } & Secondaire & 23 & 36,5 \\
\hline & Tertiaire & 11 & 17,5 \\
\hline & Quaternaire & 2 & 3,2 \\
\hline \multirow{3}{*}{ Résultat } & Hospitalisé & 0 & 0 \\
\hline & Non hospitalisé, rétabli & 57 & 100,0 \\
\hline & Décédé & 0 & 0 \\
\hline $\begin{array}{l}\text { Taux d'attaque } \\
\text { du centre } \\
\text { d'entraînement }\end{array}$ & $\begin{array}{l}\text { Cas/toutes les personnes } \\
\text { qui ont fréquenté le centre } \\
\text { d'entraînement au cours } \\
\text { des premières semaines } \\
\text { de l'enquête (du } 20 \text { au } 27 \\
\text { septembre et du } 1^{\text {er }} \text { au } 3 \\
\text { octobre) }\end{array}$ & $27 / 251$ & 10,8 \\
\hline
\end{tabular}

Figure 2 : Courbe épidémique de l'éclosion de COVID-19 associée à la fréquentation du centre d'entraînement, du 12 septembre au 20 octobre 2020, selon la date d'apparition des symptômes ou la date de prélèvement (pour les cas asymptomatiques) et l'association à un aggrégat, ville $\mathrm{X}$, Saskatchewan $(n=63)$

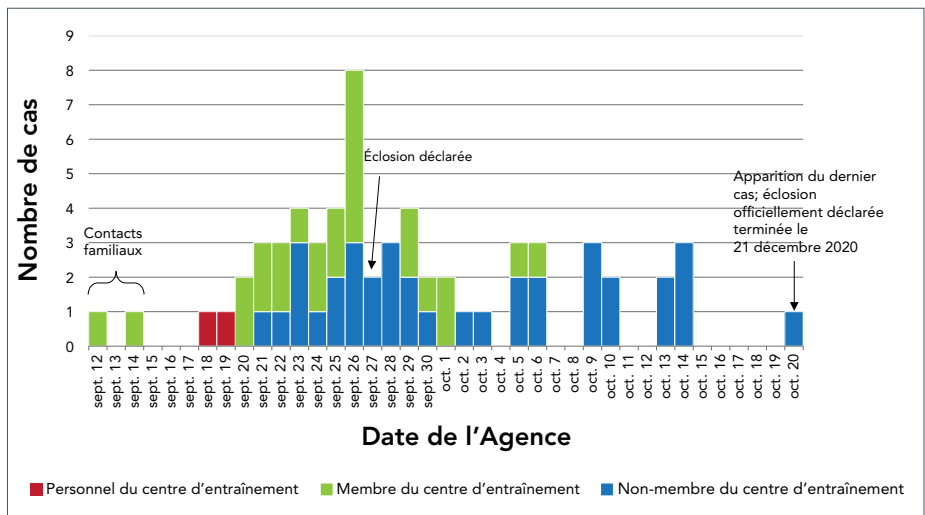

Abréviation: L'Agence, L'Agence santé publique du Canada

La date d'apparition des symptômes du cas index était le 12 septembre. Deux jours plus tard, une personne fréquentant le centre d'entraînement et un contact familial du cas index sont tombés malades. Quatre jours plus tard, une autre personne fréquentant le centre d'entraînement est tombée malade. La transmission au sein de l'établissement s'est poursuivie pendant plusieurs semaines. 
Le cas index a déclaré avoir pratiqué de l'activité physique individuelle (pas d'exercice en groupe) lorsqu'il était au centre d'entraînement et a déclaré avoir adhéré aux interventions recommandées de santé publique. Le port du masque dans les installations d'entraînement n'a été rendu obligatoire par un arrêté sanitaire provincial que le 6 novembre 2020.

Après la déclaration de l'éclosion le 27 septembre, des informations ont été recueillies auprès du centre d'entraînement sur la fréquentation au cours de la semaine précédant et suivant la déclaration de l'éclosion (du 20 septembre au 3 octobre). Le centre d'entraînement a déclaré que 251 personnes l'ont fréquenté pendant cette période, ce qui représente un taux d'attaque global d'environ $11 \%$ parmi les personnes fréquentant le centre d'entraînement $(27 / 251 ; 10,8$ \%).

Les cas directement liés au centre d'entraînement ont continué à se produire jusqu'au 20 octobre (total $n=27$ individus). Deux membres du personnel ont été infectés au cours de la période considérée et ont rapporté avoir participé principalement à des entraînements individuels (musculation et cardio).

Quatre cas qui ont fréquenté le centre d'entraînement à plusieurs reprises entre le 16 et le 24 septembre ont également fréquenté une grande école secondaire régionale $(n=590)$ alors qu'ils étaient contagieux. Malgré le dépistage actif, y compris le test d'une centaine d'élèves de l'école secondaire, il n'y a eu aucune preuve de transmission secondaire dans l'école. Ces cas ont également pris part à d'autres activités à haut risque alors qu'ils étaient infectieux à l'école, notamment en jouant au football et au hockey et en fréquentant au moins quatre groupes de classes différentes. Malgré cela, il n'y avait aucune preuve de cas supplémentaires à l'école.
Les mesures de santé publique visant à réduire la transmission du SRAS-CoV-2 à l'école ont été nombreuses. Elles comprenaient les éléments suivants : port du masque obligatoire en classe pour les élèves de $4^{\mathrm{e}}$ année et plus, y compris dans le gymnase; un horaire en « cinq blocs » où les élèves étaient placés dans de petits groupes d'apprentissage; le diner en classe uniquement; et des pauses décalées tout au long de la journée. Après la découverte des quatre cas infectieux, l'école est passée à l'apprentissage en ligne pendant environ deux semaines.

\section{Examens en laboratoire}

Les données de séquençage du génome entier pour 32 des cas liés à l'agrégat sont présentées dans la figure 3 . L'analyse des données de séquençage a permis d'attribuer les 32 spécimens à la lignée du pangolin en utilisant Pangolin v2.1.7 et les données PangoLEARN publiées le 16 janvier 2021 (Centre for Genomic Pathogen Surveillance, Royaume-Uni), avec neuf profils uniques de variants nucléotidiques simples. Le SRASCoV-2 de la lignée B.1.1 a été identifié dans le monde entier et a été fréquemment retrouvé dans des séquences ailleurs en Saskatchewan. À ce jour, le laboratoire provincial n'a pas identifié la lignée B.1.1 du SRAS-CoV-2 présentant le même profil unique de variants nucléotidiques ailleurs en Saskatchewan, mais cela n'exclut pas sa présence $(9,10)$.

\section{Enquête environnementale}

Avec la collaboration du propriétaire du centre d'entraînement, les inspecteurs de la santé publique ont travaillé pour enquêter sur la propagation virale dans le centre d'entraînement, y répondre et l'atténuer. La santé publique a fermé le centre d'entraînement le 26 septembre à $22 \mathrm{~h}$ et a autorisé sa réouverture le 30 septembre à $23 \mathrm{~h} 59$. Les inspecteurs de la santé publique ont fourni des recommandations au propriétaire (distanciation physique des clients et de l'équipement,

Figure 3 : Données de séquençage du génome entier

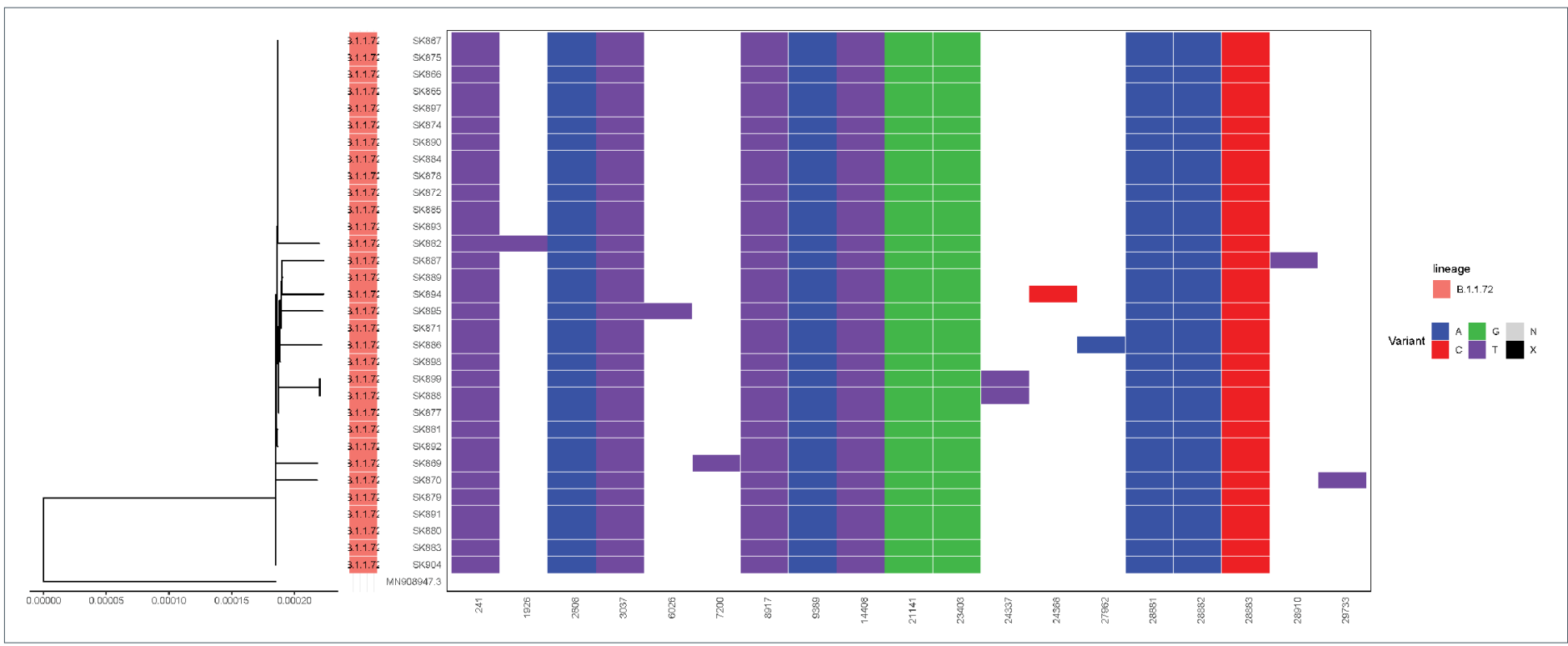

Figure en anglais seulement 
recommandations sur les ingrédients des produits de nettoyage, fréquence de nettoyage) et, conformément aux directives gouvernementales (11), ont autorisé la réouverture du centre d'entraînement après un nettoyage en profondeur de l'établissement.

Le propriétaire, lors de discussions avec l'inspecteur de la santé publique local, a indiqué que les membres du centre d'entraînement avaient respecté les recommandations en matière de distanciation physique, mais qu'ils n'avaient peutêtre pas respecté les directives relatives au port du masque (l'obligation provinciale de porter un masque n'était pas en vigueur à l'époque). L'inspecteur a noté que les équipements du centre d'entraînement n'étaient pas tous espacés de deux mètres; certains équipements d'entraînement cardio ont été déplacés afin de respecter les règles d'éloignement. La désinfection du gymnase au moyen d'un pulvérisateur électrostatique aurait eu lieu; cependant, on ne sait pas avec certitude si elle était systématique ou si elle était fréquente.

L'inspection du système de ventilation de l'air a permis de découvrir deux parties principales : le système de la zone principale du centre d'entraînement a été installé en 2013, tandis que le système de la deuxième zone, plus petite, a été installé en 2019. L'inspection a permis de constater que le système de traitement de l'air était adéquat pour une bonne ventilation; cependant, les mesures réelles du débit et de l'échange d'air n'ont pas été prises. Le propriétaire du centre d'entraînement déclaré qu'il changeait les filtres à air toutes les deux semaines. L'inspecteur de la santé publique a indiqué que la ventilation du centre d'entraînement pourrait avoir joué un rôle dans l'éclosion : ce centre d'entraînement particulier est situé dans un sous-sol à plafond bas.

\section{Discussion}

Une éclosion de COVID-19 a été associée à la fréquentation d'un centre d'entraînement dans une ville canadienne. Un total de 63 cas liés à l'éclosion, confirmés par le séquençage du génome entier, ont été identifiés avec des dates d'apparition allant du 12 septembre au 20 octobre 2020; tous les cas se sont rétablis. Bien que des cas liés à l'éclosion aient fréquenté l'école alors qu'ils étaient infectieux et malgré le dépistage actif, y compris le dépistage d'une centaine d'élèves à l'école secondaire, il n'y a eu aucune preuve de transmission secondaire au sein de l'école. Dans cette éclosion, la fréquentation d'un centre d'entraînement n'a pas donné lieu à un événement ponctuel de supercontamination. Comme une cohorte commune fréquente les centres d'entraînement au fil du temps, nous supposons que la fermeture à court terme et le nettoyage peuvent ne pas être suffisants pour interrompre les chaînes de transmission dans les centres d'entraînement.
Les enquêtes de santé publique, y compris les enquêtes approfondies de cas et le dépistage actif par des infirmières de santé publique formées, la recherche des contacts, l'isolement des personnes exposées, l'inspection, l'éducation, la surveillance et les tests, ont été combinés pour contrôler la propagation de la COVID-19. Bien que les cas du centre d'entraînement aient également suivi des cours dans une école secondaire et participé à des activités sportives alors qu'ils étaient infectieux, aucune transmission virale n'a eu lieu au sein de l'école.

Au moment où les cas infectieux fréquentaient l'école, celle-ci avait mis en place de nombreuses mesures de santé publique destinées à réduire la transmission virale. L'hypothèse est que ces mesures ont réduit la propagation à l'intérieur de l'école, en combinaison avec les preuves croissantes que les enfants sont des transmetteurs viraux moins efficaces lorsqu'ils sont infectés par un virus de type sauvage $(12,13)$, bien que ce raisonnement puisse être moins applicable aux adolescents plus âgés.

Dans cette enquête sur la propagation de l'éclosion, nous avons constaté que la fréquentation du centre d'entraînement n'a pas donné lieu à un événement ponctuel de supercontamination. Les dates d'apparition des symptômes chez les personnes fréquentant le centre d'entraînement étaient étalées dans le temps (figure 2), ce qui indique une cohorte de personnes fréquentant le centre d'entraînement qui s'entremêlent à différents moments. En raison de la nature de la fréquentation continue du centre d'entraînement au fil du temps, la fermeture à court terme et le nettoyage, comme le recommandent les directives gouvernementales pour les centres d'entraînement et les salles de sport (11), n'interrompront pas nécessairement les chaînes de transmission.

Les rapports d'éclosion antérieurs $(1,3,4)$ ont identifié les centres d'entraînement et les salles de sport comme des lieux à haut risque pour la transmission du SRAS-CoV-2. Cependant, en dehors des rapports d'éclosion, les études visant à quantifier le risque d'infection associé à la communauté dans les centres d'entraînement n'ont pas uniformément démontré une augmentation du risque. En l'absence d'un cas infectieux lié à une installation, comme c'était le cas dans l'étude de Helsingen et al. (7), il n'est pas surprenant que les taux de positivité des tests parmi les personnes fréquentant les centres d'entraînement et celles qui ne les fréquentent pas soient restés faibles. Une étude de cas-témoins menée par les Centers for Disease Control and Prevention (CDC) n'incluait que des individus symptomatiques, c'est-à-dire que ceux qui avaient eu des résultats de test négatifs étaient des témoins et ceux qui étaient positifs constituaient des cas (8). Étant donné que les auteurs de l'étude n'ont recruté que des personnes symptomatiques positives au moment du test, il est possible qu'un biais de sélection systématique se soit produit. Les personnes asymptomatiques et infectieuses (non échantillonnées dans l'étude) peuvent avoir été plus susceptibles de se rendre 
dans un centre d'entraînement avant le test comparativement aux personnes symptomatiques (population étudiée) au moment du test, qui peuvent avoir été moins susceptibles de se rendre dans un centre d'entraînement avant le test si elles ne se sentaient pas bien. Ce biais peut avoir diminué artificiellement le risque associé à ce type de milieu parmi les participants à l'étude.

Les enquêtes par aggrégats sont importantes pour comprendre la dynamique de transmission des agents pathogènes. Les enquêtes de santé publique ont démontré à plusieurs reprises qu'il existe un risque d'infection par le SRAS-CoV-2 dans les centres d'entraînement. Dans l'aggrégat décrit dans cet article, nous émettons l'hypothèse que les facteurs suivants ont probablement contribué à la transmission virale dans l'établissement : le non-respect de certaines mesures de santé publique recommandées; le fait que l'éclosion se soit produite avant l'obligation provinciale de porter un masque dans les centres d'entraînement; et les plafonds bas et l'emplacement dans un sous-sol avec une ventilation potentiellement inadéquate.

Notre étude a des limites. Nous n'avons pas été en mesure de tester tous les contacts proches (les enquêteurs estiment que nous avons testé environ $70 \%$ de ceux-ci). Une liste complète des membres du centre d'entraînement n'était pas disponible, et les contacts nommés n'ont pas tous accepté de passer un test ou n'ont pas été réceptifs au suivi de la santé publique. En outre, un questionnaire spécifique aux établissements d'entraînement physique n'a pas été fourni à tous les cas impliqués dans l'aggrégat en raison de la charge de travail de la santé publique. Cela limite notre capacité à quantifier l'incidence d'autres variables qui peuvent avoir de l'importance, comme le temps passé au gymnase et le nombre de visites dans l'établissement pendant la période en question.

Il est probable que le risque accru d'infection par le SRAS-CoV-2 associée à la fréquentation d'un centre d'entraînement soit multifactoriel. La fréquentation d'un centre d'entraînement, de par sa nature même, ne se produit pas seulement à un moment donné dans le temps et l'espace (générant des événements de supercontamination); le risque d'infection est plutôt associé à une exposition continue parmi les membres du centre d'entraînement. Dans ce cas, les mesures de santé publique consistant à fermer et à nettoyer les installations d'entraînement n'ont peut-être pas été suffisantes. II pourrait être utile de cibler les centres d'entraînement pour réduire la transmission, par exemple en procédant à une inspection proactive des systèmes de ventilation, en exigeant que les registres de clients (liste de toutes les personnes fréquentant le centre d'entraînement) soient soumis à la santé publique, en utilisant des technologies, telles que les codes $\mathrm{QR}$, pour effectuer un suivi des clients, en restreignant les activités dans les centres d'entraînement connues pour être plus risquées (cours de cardiovélo, entraînement en groupe), et en mettant en œuvre un dépistage actif des cas et un dépistage rapide au point de service, en particulier parmi le personnel et les instructeurs pour qui le risque de transmission est plus élevé que celui des membres (3).

\section{Conclusion}

Les installations d'entraînement à l'intérieur sont des lieux à haut risque pour la transmission virale du SRAS-CoV-2. Le dépistage actif de cas à l'aide de trousses de test rapide au point de service dans les centres d'entraînement ou le dépistage routinier de tous les membres du centre d'entraînement lorsque les taux de transmission communautaire sont élevés peuvent constituer des stratégies efficaces à envisager dans les milieux à haut risque. À mesure que la vaccination contre l'infection par le SRAS-CoV-2 devient systématiquement disponible au niveau de la population, le statut vaccinal peut être un élément d'information utile à recueillir dans ces milieux à haut risque. Il est probable que les personnes vaccinées émettront moins de virus; cependant, le respect des mesures de santé publique (telles que le nettoyage en profondeur, la désinfection du matériel, la distanciation physique, la réduction des activités d'entraînement en groupe et des limites de capacité) et l'utilisation de masques lorsque la ventilation est mauvaise resteront importants. Cette recommandation peut s'étendre à d'autres cadres tels que les lieux de culte et autres lieux de rassemblement.

\section{Déclaration des auteurs}

E. H., A. L., R. M. et M. T. — Ont effectué la collecte des données

M. T., A. C., J. M., R. M. et M. A. - Ont préparé le matériel et effectué l'analyse

M. A. - A rédigé le manuscrit

Tous les auteurs ont contribué à la conception et l'élaboration de l'étude, à l'interprétation, ont commenté les ébauches et contribué à la formulation, ont lu et approuvé le manuscrit final.

Le contenu de l'article et les points de vue qui y sont exprimés n'engagent que les auteurs et ne correspondent pas nécessairement à ceux du gouvernement du Canada.

\section{Intérêts concurrents}

Les auteurs déclarent qu'ils n'ont pas de conflit d'intérêts.

\section{Financement}

Les auteurs remercient la Saskatchewan Health Authority, Population Health et le laboratoire provincial Roy Romanow pour leur appui non financier. 


\section{Références}

1. Bae $S$, Kim H, Jung TY, Lim JA, Jo DH, Kang GS, Jeong SH, Choi DK, Kim HJ, Cheon YH, Chun MK, Kim M, Choi S, Chun C, Shin SH, Kim HK, Park YJ, Park O, Kwon HJ. Epidemiological characteristics of COVID-19 outbreak at fitness centers in Cheonan, Korea. J Korean Med Sci 2020;35(31):e288. DOl PubMed

2. Pung R, Chiew CJ, Young BE, Chin S, Chen MI, Clapham HE, Cook AR, Maurer-Stroh S, Toh MP, Poh C, Low M, Lum J, Koh VT, Mak TM, Cui L, Lin RV, Heng D, Leo YS, Lye DC, Lee VJ; Singapore 2019 Novel Coronavirus Outbreak Research Team. Investigation of three clusters of COVID-19 in Singapore: implications for surveillance and response measures. Lancet 2020;395(10229):1039-46. DOI PubMed

3. Jang S, Han SH, Rhee JY. Cluster of coronavirus disease associated with fitness dance classes, South Korea. Emerg Infect Dis 2020;26(8):1917-20. DOI PubMed

4. National Collaborating Centre for Methods and Tools. Rapid review: What is known about the risk of COVID-19 transmission across different indoor settings in the community such as restaurants and gyms? Hamilton (ON): NCCMT; 2020. https://www.nccmt.ca/covid-19/covid-19rapid-evidence-service/30

5. Furuse $Y$, Sando E, Tsuchiya N, Miyahara R, Yasuda I, Ko YK, Saito M, Morimoto K, Imamura T, Shobugawa Y, Nagata $S$, Jindai K, Imamura T, Sunagawa T, Suzuki M, Nishiura $\mathrm{H}$, Oshitani $\mathrm{H}$. Clusters of coronavirus disease in communities, Japan, January-April 2020. Emerg Infect Dis 2020;26(9):2176-9. DOI PubMed

6. Buonanno G, Morawska L, Stabile L. Quantitative assessment of the risk of airborne transmission of SARS-CoV-2 infection: prospective and retrospective applications. Environ Int 2020;145:106112. DOI PubMed

7. Helsingen LM, Løberg M, Refsum E, Gjøstein DK, Paulina Wieszczy P, Olsvik $\varnothing$, Juul FE, Barua I, Jodal HC, Herfindal M, Mori Y, Jore S, Lund-Johansen F, Fretheim A, Bretthauer M, Kalager M; The TRAiN study group. A Randomised Trial of Covid-19 Transmission in Training Facilities. medRxiv 2020.06.24.20138768 [Pré-impression]. Posted 2020-11-17 (accédé 2020-11-24). DOI
8. Fisher KA, Tenforde MW, Feldstein LR, Lindsell CJ, Shapiro NI, Files DC, Gibbs KW, Erickson HL, Prekker ME, Steingrub JS, Exline MC, Henning DJ, Wilson JG, Brown SM, Peltan ID, Rice TW, Hager DN, Ginde AA, Talbot HK, Casey JD, Grijalva CG, Flannery B, Patel MM, Self WH; IVY Network Investigators; CDC COVID-19 Response Team. CDC COVID-19 Response Team. Community and close contact exposures associated with COVID-19 among symptomatic adults $\geq 18$ years in 11 outpatient health care facilities- united States, July 2020. MMWR Morb Mortal Wkly Rep 2020 Sep;69(36):1258-64. DOI PubMed

9. GISAID. Lineage B.1.1.72 [Internet]. Hixton (UK): Centre for Genomic Pathogen Surveillance; 2020 (accédé 2020-11-24). https://cov-lineages.org/lineages/lineage_B.1.1.72.html

10. GISAID. cov-lineages/pangolin [Internet]. Hixton (UK): Centre for Genomic Pathogen Surveillance; 2021 (accédé 2021-02-17). https://github.com/cov-lineages/pangolin

11. Government of Saskatchewan. Gyms and fitness facilities guidelines. Guidelines for the re-open Saskatchewan plan [Internet]. Regina (SK): Government of Saskatchewan; 2020 (accédé 2020-11-15). https://www.saskatchewan. $\mathrm{ca} /$ government/health-care-administration-and-providerresources/treatment-procedures-and-guidelines/emergingpublic-health-issues/2019-novel-coronavirus/re-opensaskatchewan-plan/guidelines/gyms-and-fitness-facilitiesguidelines

12. Isaacs $D$, Britton $P$, Howard-Jones $A$, Kesson A, Khatami A, Marais B, Nayda C, Outhred A. To what extent do children transmit SARS-CoV-2 virus? J Paediatr Child Health 2020;56(6):978-9. DOI PubMed

13. Riediker M, Morawska L. Low exhaled breath droplet formation may explain why children are poor SARS-CoV-2 transmitters. Aerosol Air Qual Res 2020;20(7):1513-5. DOI 